\title{
Decay ring status / studies
}

\author{
ANTOINE CHANCE*广 \\ CEA, IRFU, SACM, Centre de Saclay, F-91191 Gif-sur-Yvette, France. \\ E-mail: achancedcea.fr
}

\begin{abstract}
The aim of "beta-beams" is to produce highly energetic pure electron neutrino and anti-neutrino beams coming from $\beta$-decay of radioactive ions. In CERN baseline, after accelerating, the ions ${ }^{6} \mathrm{He}^{2+}$ and ${ }^{18} \mathrm{Ne}^{10+}$ are stored in a racetrack-shaped-decay ring until they are lost [1]. Consequently, the injection compensates the losses which occurred between two cycles. Two main loss sources were identified: the $\beta$ decay and the injection scheme. After giving the optics, we will see how to protect the magnetic elements from the decay products. The injections scheme will be then detailed with its implications. We will see that the injection process makes a collimation section in energy necessary. Since the magnetic elements are not perfect, we will take into account the magnet misalignment and the multipole defects in the dipoles. We will talk then about the closed orbit distortion due to misalignment defects and about the long-term transverse stability with the dynamic aperture.
\end{abstract}

10th International Workshop on Neutrino Factories, Super beams and Beta beams June 30 - July 52008

Valencia, Spain

\footnotetext{
* Speaker.

${ }^{\dagger}$ Work supported by European Community under the FP6 - Research Infrastructure Action - Structuring the European Research Area - EURISOL DS Project Contract no. 515768 RIDS.
} 


\section{Optics}

The decay ring is a $6911.5 \mathrm{~m}$-long racetrack-shaped-ring with two 2468 -m-long straight sections, of which one is directed toward the detector situated in the Fréjus tunnel [1]. The useful section is around $36 \%$ of the circumference. The parameters of the beam are given in Table 1 [ [2].

Table 1: Nominal parameters for the decay ring.

\begin{tabular}{|c|c|c|c|}
\hline & Units & ${ }^{6} \mathrm{He}^{2+}$ & ${ }^{18} \mathrm{Ne}^{10+}$ \\
\hline$\gamma$ & - & 100 & 100 \\
\hline$B \rho$ & T.m & 938 & 563 \\
\hline$B_{\text {dipole }}$ & $\mathrm{T}$ & 6 & 3.6 \\
\hline$\tau$ at rest & $\mathrm{s}$ & 0.8 & 1.67 \\
\hline$N_{\text {injected }}$ (ions/batch) & - & $9.05 \times 10^{12}$ & $4.26 \times 10^{12}$ \\
\hline$N_{\text {stored }}$ (ions/batch) & - & $9.71 \times 10^{13}$ & $7.40 \times 10^{13}$ \\
\hline Harm. number & - & 924 & 924 \\
\hline Bunch number & - & 20 & 20 \\
\hline rms $\varepsilon_{x}$ & $\pi$ mm.mrad & 0.11 & 0.11 \\
\hline rms $\varepsilon_{y}$ & $\pi$ mm.mrad & 0.06 & 0.06 \\
\hline$\Delta v_{x}$ & - & -0.015 & -0.127 \\
\hline$\Delta v_{y}$ & - & -0.024 & -0.201 \\
\hline
\end{tabular}

A first consequence to store high intensity beams is that the tune spread due to the space charge effects may not be negligible. The tune shift was calculated for ${ }^{6} \mathrm{He}^{2+}$ and ${ }^{18} \mathrm{Ne}^{10+}$ and is given in Table 1. With nominal parameters, the space charge effects can be neglected in ${ }^{6} \mathrm{He}^{2+}$ case contrary to ${ }^{18} \mathrm{Ne}^{10+}$ case. A solution to decrease the space charge effect would be to increase the rms emittance of the stored beam by injecting a mismatched beam. To have a negligible tune shift for ${ }^{18} \mathrm{Ne}^{10+}$ of -0.063 in the horizontal plane and -0.055 in the vertical one, the rms emittance must then be enlarged up to $0.22 \pi \mathrm{mm}$.mrad in both planes. In the following, we will assume this rms emittance for ${ }^{18} \mathrm{Ne}^{10+}$ to neglect space charge effects.

The arcs are $2 \pi$ insertions of which the optics is given on Figure 11. Four functional parts were distinguished in the arcs [3]: a regular FODO lattice in the arc, a matching section between the long straight section and the FODO lattice, which is used to extract the decay products coming from the long straight section too, a low $\beta$ high dispersion insertion for the injection and a matching section between the regular FODO lattice and the insertion. Moreover, large betatron functions are needed in the FODO lattices of the long straight sections to maximize the neutrino flux going to the detector.

\section{Losses by $\beta$ decay}

The decay of the stored ions implies a continuous power loss with a mean value of $10.8 \mathrm{~W} / \mathrm{m}$ for ${ }^{6} \mathrm{He}^{2+}$ and $11.8 \mathrm{~W} / \mathrm{m}$ for ${ }^{18} \mathrm{Ne}^{10+}[$ [ $]$. The relative rigidity difference between the decay product and the reference one is then around $-33 \%$ for ${ }^{6} \mathrm{He}^{2+}$ and $+11 \%$ for ${ }^{18} \mathrm{Ne}^{10+}$, which is too large to 

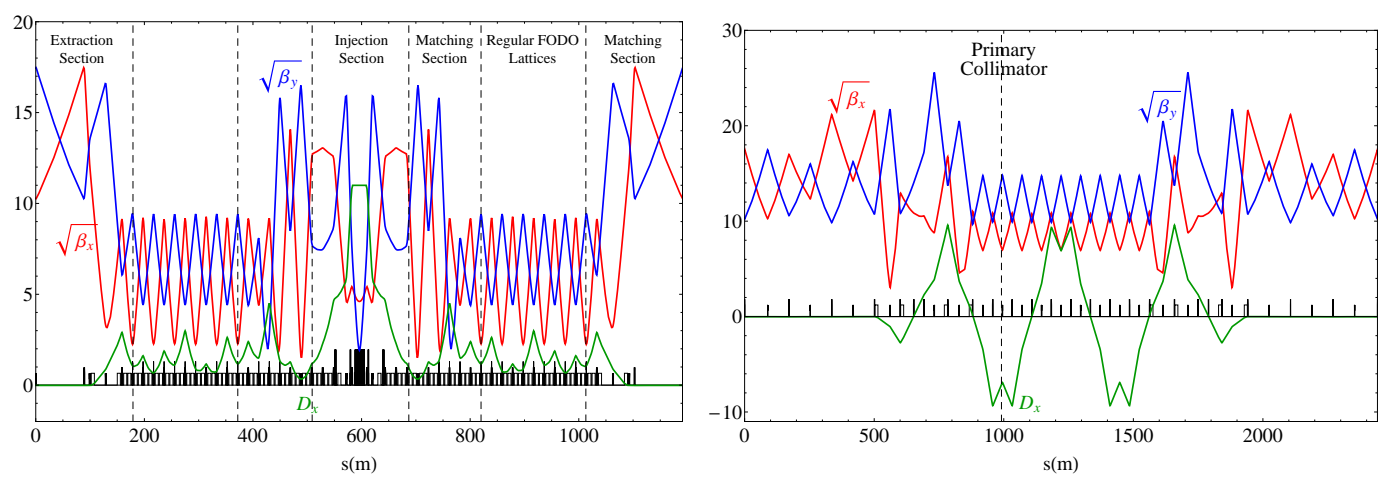

Figure 1: Optical Functions in the arc (left) and in the collimation section (right).

accept the decay products. They are then lost after crossing a few dipoles. Two principal problematics can be underlined. After the long straight sections, the deposition is equal to several tens of kilowatts: a dedicated extraction section at the arc entry is needed. Moreover, the deposition inside the superconducting magnets must be low enough to avoid quenching, for which two solutions were studied. The first one consists in inserting absorbers between the magnetic elements. The second one consists in using open mid plane dipoles, which are dipoles without coils in the horizontal plane. The advantages of this solution is to enable longer dipoles, which decreases their magnetic field ( $5 \mathrm{~T}$ instead of $6 \mathrm{~T}$ ), to reduce the needed dipole aperture ( $\pm 50 \mathrm{~mm}$ against $\pm 80 \mathrm{~mm}$ ) and to make the structure changes simpler if other ions are used.

To evaluate the deposition of the decay products in the chamber, a simple 1D program was implemented in BETA [\$]. It does not take into account any interaction with the walls. To improve the model, a 3D simulation with the interactions was run in the case of absorbers [ [6]. In both simulations, the average value deposited in the dipoles is then less than $10 \mathrm{~W} / \mathrm{m}$. Unfortunately, peaks exceed recommended limit: $4.3 \mathrm{~mW} / \mathrm{cm}^{3}$ for dipoles. The 3D simulation with open mid plane dipoles has not been performed yet.

\section{Collimation in energy}

The injection system relies on injecting a fresh beam in a dispersive area at an energy different from the reference one. The stored beam and the injected one are then horizontally separated. The injection can be cut in four main steps. Firstly, the stored beam is deviated by four kickers. Secondly, the entering beam is injected "off momentum" at $\delta=5 \times 10^{-3}$ on its chromatic orbit. Thirdly, the kickers are switched off. The injected beam stays on its chromatic orbit and runs under the septum blade. Finally, both beams are merged together by a specific RF program using two variable cavity families, of which one is at double frequency [7].

Several injection cycles were simulated, which has identified two loss sources. The first one comes from the fresh ions which are not captured at the injection. The second one is due to the blow-up in the space $(l, \delta)$ injection after injection. On Figure 2 , the survival of a beam injected at $t=0$ is represented as a function of the number of injection cycles. After around 15-20 injections, most ions are not accepted anymore. When the steady state is reached, the loss amount between two injections is compensated by the injection itself. Around $50 \%$ (Helium) and $20 \%$ (Neon) of 
the losses which occurred between two cycles are due to the $\beta$ decay. The mean power to collimate in the ring can then be evaluated to $74 \mathrm{~kW}$ for ${ }^{6} \mathrm{He}^{2+}$ and $248 \mathrm{~kW}$ for ${ }^{18} \mathrm{Ne}^{10+}$. Therefore, a two-step collimation system is needed and was designed to collimate in energy at $\delta=2.5 \times 10^{-3}$ ( Figure 1). It is located in the long straight section which is not directed toward the detector.
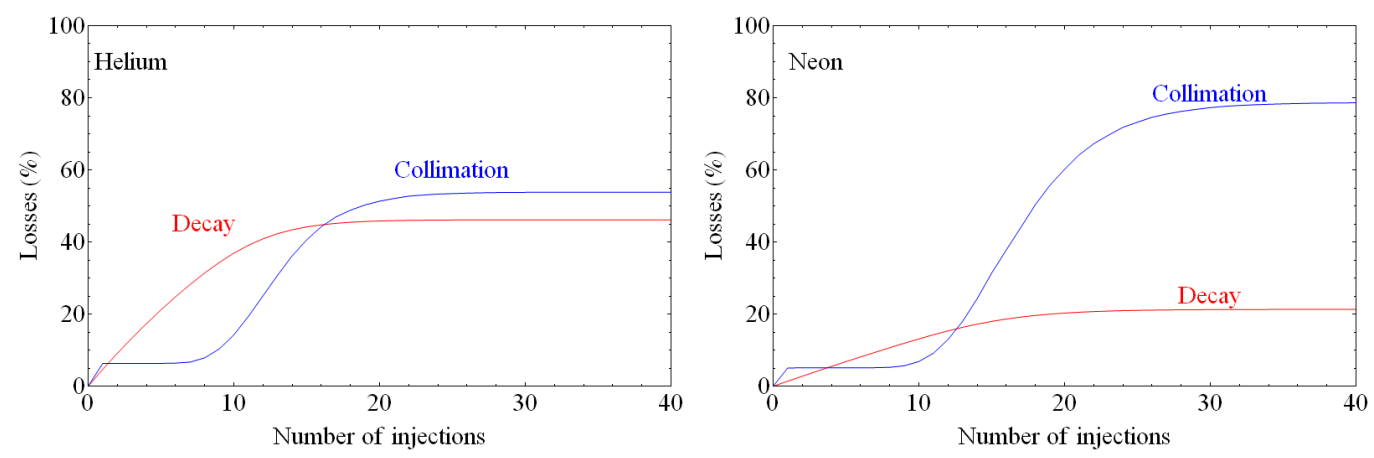

Figure 2: Loss amount as a time function. At $t=0$, the beam has just been injected. In red, decay losses and in red, losses due to the collimation in energy for Helium (left) and Neon (right).

\section{Defects in the magnetic elements}

Misalignments and errors on the main field are to take into account in the magnetic elements. One of their consequences is a distortion of the closed orbit. The random rms errors on the magnetic field, the rms transverse misalignment and the rotation around the machine axis are respectively assumed to be $0.5 \times 10^{-3}, 0.5 \mathrm{~mm}$ and $1 \mathrm{mrad}$ for the dipoles. The rms error on the gradient in the quadrupoles is assumed to be $1 \times 10^{-3}$. Their rms transverse misalignment is $0.4 \mathrm{~mm}$. The BPMs are assumed to be ideal. The order of magnitude of the closed orbit distortion is then a few centimeters, which makes the closed orbit correction necessary. 83/85 horizontal/vertical BPMs were inserted in the structure. After correction, the rms resultant of the closed orbit is then less than $0.4 \mathrm{~mm}$. The maximum rms value for the horizontal dipole correctors is $0.042 \mathrm{mrad}$ and for the vertical ones $0.064 \mathrm{mrad}$. Since the closed orbit is corrected up to three standard deviations, the integrated field in the dipole correctors must be respectively 0.118 T.m and 0.180 T.m.

The natural chromaticity in the decay ring is respectively -1.72 and -2.35 in each plane. Without correction, the injected beam is not accepted. After correcting the chromaticity with only two sextupole families, we obtain the dynamic aperture of the blue curve on Figure 3. The dynamic aperture is quite large but the coupling resonances are not corrected and the beam does not keep an elliptic shape. After minimizing the sextupolar resonances with six sextupole families, we obtain the red curve on Figure 3 . The dynamic aperture is much larger.

However, the obtained dynamic aperture is in the case of ideal dipole magnets. The large aperture of the dipoles implies unavoidable multipole components. Until now, only systematic multipole components were considered. The assumed systematic multipole components in the dipoles are $b_{3}=-1.68 \times 10^{-4}, b_{5}=33.02 \times 10^{-4}, b_{7}=-50.12 \times 10^{-4}$ and $b_{9}=29.58 \times 10^{-4}$ at the reference radius $60 \mathrm{~mm}[8]$. A direct consequence is a reduction of the dynamic aperture. In order to identify the main multipole effects, the Figure 3 gives the dynamic aperture calculated 
for each alone multipole component for the same reference structure. The strongest contribution comes from the fifth and the seventh order multipole components. In order to decrease their effects, different working points were considered and an automatic enlargement program of the dynamic aperture was added in BETA, which enabled to obtain a dynamic aperture of more than $6 \sigma$ [9].
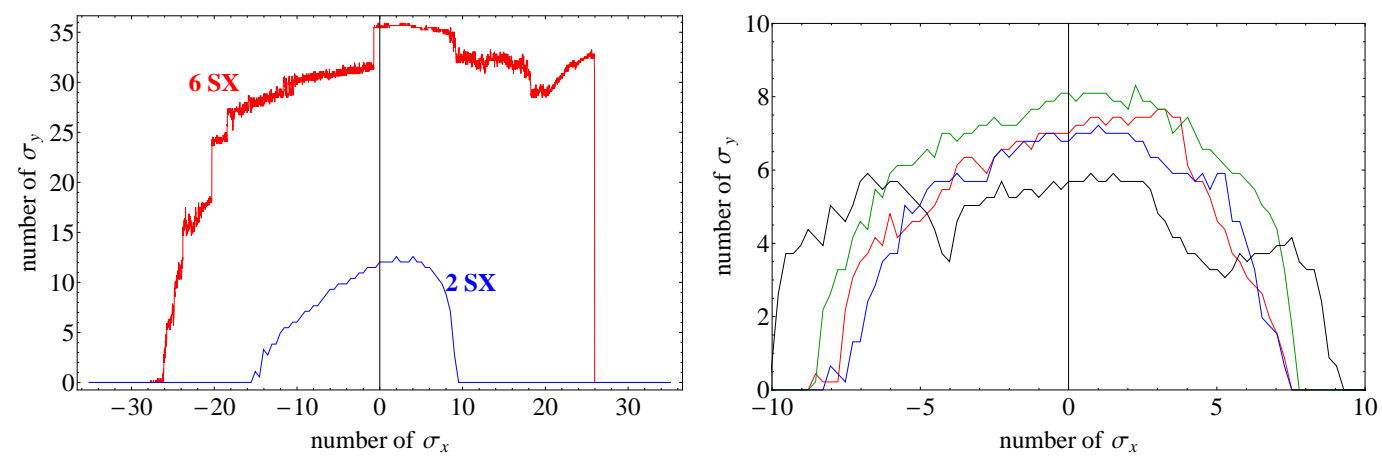

Figure 3: Dynamic aperture for 1000 turns. On left, the dipoles are ideal and in blue, 2 sextupole families were used and in red 6 sextupole families. On right, the multipole defects of the fifth order (red), the seventh order (blue), the ninth order (green) or every multipole component (black) were applied.

\section{Conclusion}

The optics of the decay ring has been given at first and second orders. Some problematics have been studied as the decay losses, the injection system, the collimation in energy or the errors in the dipoles. In a near future, the transport of the beam in the collimation section should be simulated. The interaction with the walls and the $6 \mathrm{D}$ beam distribution must be taken into account. The effect of the high intensities stored on the beam dynamics (beam loading, space charge, impedances, ...) is to study too.

\section{References}

[1] M. Benedikt et al., Baseline Design for a Beta-Beam Neutrino Facility, in proceedings of EPAC'O4.

[2] Parameter and Intensity Values, Version 2, July 2005, EURISOL DS/TASK12/TN-05-03.

[3] A. Chancé, Etude et conception de l'anneau de désintégration d'une usine à neutrinos utilisant les décroissances beta des noyaux Hélium 6 et Néon 18 produits par un faisceau intense de protons frappant diverses cibles, $\mathrm{PhD}$ thesis, Université Paris Sud, 2007.

[4] A. Chancé et al., Loss Management in the Beta-Beam Decay Ring, in proceedings of EPAC'06.

[5] J. Payet, Code BETA, LNS Version.

[6] F.W. Jones et al., Simulation of Decays and Secondary Ion Losses in a Betabeam Decay Ring, in proceedings of $P A C^{\prime} 07$.

[7] S. Hancock et al., Stacking Simulations in the Beta-Beam Decay Ring, in proceedings of EPAC'O6.

[8] C. Vollinger et al., private communication, 2006.

[9] A. Chancé et al., Studies of dipole field quality for the beta-beam decay ring, in proceedings of PAC'07. 